\title{
Prognostic factors of acute heart failure in patients with pulmonary arterial hypertension
}

\author{
B. Sztrymf, R. Souza, L. Bertoletti, X. Jaïs, O. Sitbon, L.C. Price, \\ G. Simonneau and M. Humbert
}

ABSTRACT: Acute right ventricular failure in the setting of pulmonary arterial hypertension (PAH) often requires hospitalisation in intensive care units (ICU) to manage the subsequent low cardiac output and its consequences. There are very few data on these acute events.

We recorded demographic, clinical and biological data and therapy in consecutive patients suffering from acute right heart failure requiring catecholamine treatment in the ICU of the French referral centre for pulmonary hypertension. These variables were analysed according to the survival status in ICU.

46 patients were included, the mean age was 50.3 yrs. ICU mortality was $41 \%$. We found no difference in terms of demographics, clinical data, last haemodynamic measurements at admission. Systemic arterial pressure was significantly lower in the subgroup of patients whose clinical course was fatal. Plasma brain natriuretic peptide (BNP), C-reactive protein (CRP), serum sodium and creatinine at admission correlated with survival. Demonstration of an infection during the ICU stay was associated with a worse prognosis.

These preliminary results underline the importance of some simple clinical and biological parameters in the prognostic evaluation of acute heart failure in the setting of PAH. Whether these parameters can guide therapy needs to be further investigated.

KEYWORDS: Brain natriuretic peptide, heart failure, intensive care unit, pulmonary arterial hypertension, survival

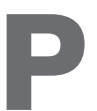
ulmonary arterial hypertension $(\mathrm{PAH})$ is a disease affecting small pulmonary arteries, with progressive vascular narrowing leading to elevated pulmonary arterial resistance and right ventricular failure [1]. PAH patients may experience acute right ventricular (RV) failure during the course of their disease [2]. These episodes often require hospitalisation in an intensive care unit (ICU) because of the requirement for close monitoring and for vasoactive drugs. Many studies of acute RV failure in the absence of previous pulmonary hypertension (mainly in severe pulmonary embolism) have been performed and some guidelines are available in that setting [3]. To the best of our knowledge, no study has yet prospectively investigated cases of severe RV failure in PAH patients. Although there are undoubtedly some similarities with other causes of acute RV failure, the physiological mechanisms may differ in $\mathrm{PAH}$ patients where the RV has adapted to the chronically elevated afterload, compared to patients with, for example, an acute pulmonary

For editorial comments see page 1203. embolism where the RV is relatively "naïve" [4]. In the absence of guidelines for treatment in this specific patient population, current therapeutic endpoints are extrapolated from other aetiologies of RV failure, with the use of appropriate fluid balance, inotropic and vasoconstrictive agents (including catecholamines) to maintain adequate aortic root and right coronary artery perfusion pressure to maintain cardiac output and tissue dobutamine because of its positive inotropic effects and beneficial RV-pulmonary artery coupling [7] as well as its ability to exert minimal tachycardia compared with dopamine [8]. Norepinephrine has been reported to be used alone or in combination with dobutamine in this setting, and experimental data support its usefulness in a model of RV pressure overload [9]. Neither ICU outcome following decompensated $\mathrm{PAH}$, leading to severe RV failure, nor the impact of one of these episodes on baseline disease course in survivors is known. In an attempt to answer these questions and to describe the oxygenation $[5,6]$. Some data support the use of
AFFILIATIONS

Service de Pneumologie et

Réanimation Respiratoire, Centre des Maladies Vasculaires Pulmonaires, UPRES EA 2705, Hôpital Antoine Béclère, Assistance Publique, Hôpitaux de Paris; Université Paris Sud, Clamart, France.

CORRESPONDENCE B. Sztrymf

Service de Pneumologie et Réanimation Respiratoire Hôpital Antoine Béclère 157 rue de la Porte de Trivaux 92140 Clamart

France

E-mail: benjamin.sztrymf@ abc.aphp.fr

Received:

April 302009

Accepted after revision:

Oct 162009

First published online: Nov 062009 
clinical characteristics of this severe subset of patients, we performed a single-centre prospective study of consecutive patients admitted to our ICU (National Reference Centre for Pulmonary Hypertension, Hôpital Antoine Béclère, Assistance Publique, Hôpitaux de Paris; Université Paris Sud, Clamart, France).

\section{METHODS}

\section{Design}

This was a prospective single-centre study involving consecutive patients.

\section{Patients}

All patients with chronic pre-capillary pulmonary hypertension (inoperable chronic thromboembolic disease and $\mathrm{PAH}$ ) referred to the ICU of the National Reference Centre for Pulmonary Hypertension were eligible. Patients were enrolled if they exhibited an acute deterioration of their condition (within 7 days before ICU admission) and required treatment with catecholamines because of clinical features of low cardiac output and/or severe RV failure All patients had been previously diagnosed with pre-capillary pulmonary hypertension according to current international guidelines (mean pulmonary artery pressure $\geqslant 25 \mathrm{mmHg}$ and pulmonary artery wedge pressure $\leqslant 15 \mathrm{mmHg}$ at rest during right heart catheterisation). The study period began in November 2005 and ended in April 2007. 46 consecutive patients were studied during their first ICU admission, and repeated admissions were not included. All patients gave informed consent to be entered in this analysis which was set up in agreements with the Commission Nationale de l'Informatique et des Libertés, the organisation dedicated to information technology and civil rights in France.

\section{Management in the ICU}

Dobutamine is first-line therapy in these patients with decompensated $\mathrm{PAH}$, with further addition of norepinephrine for persistent systemic hypotension as needed to maintain normotension and improve right coronary arterial perfusion pressure. In practice, the infusion of dobutamine is commenced at $5 \mu \mathrm{g} \cdot \mathrm{kg}^{-1} \cdot \mathrm{min}^{-1}$, with an evaluation period of $4-6 \mathrm{~h}$, after which, the infusion rate is increased by $5 \mu \mathrm{g} \cdot \mathrm{kg}^{-1} \cdot \mathrm{min}^{-1}$ every $4-6 \mathrm{~h}$ to a maximum of $20 \mu \mathrm{g} \cdot \mathrm{kg}^{-1} \cdot \mathrm{min}^{-1}$, these changes are made according to clinical assessment of low cardiac output and/or RV failure, noting that dobutamine itself may, of course, reduce blood pressure due to B2 effects. No further increment was made if systemic arterial pressure remained stable. If persistent low systemic pressure occurred, norepinephrine was added and progressively increased by $0.5 \mathrm{mg} \cdot \mathrm{h}^{-1}$ every $2 \mathrm{~h}$ until systemic arterial pressure was restored. This approach was modified depending on the aetiology of the acute worsening and on further associated haemodynamic challenges, such as sepsis. Exact fluid balance and intravenous diuretic doses are not guided by a treatment protocol but are left at the discretion of the attending physicians, as is initiation of antibiotic therapy in cases of documented or suspected infection. Due to the potentially deleterious haemodynamic consequences of positive intrathoracic pressure on RV function [10], no patient underwent mechanical ventilation in the study period. Our therapeutic approach is based on generally accepted goals in the management of acute right heart failure
[5] and our group have recently discussed endpoints in acute heart failure in $\mathrm{PAH}$ patients [11].

\section{Data}

Baseline demographic data, New York Heart Association (NYHA) status and right heart haemodynamics when last stable before admission were recorded. We analysed clinical data (evidence of a triggering factor or an infection, systemic arterial pressure, heart rate, diuresis, treatments and dose regimen) and biological data (serum level of creatinine, sodium, brain natriuretic peptide (BNP), C-reactive protein $(\mathrm{CRP})$, troponin Ic) during the stay in ICU, from the day of admission to discharge or death. The primary endpoint of the study was ICU survival. We also monitored simplified acute physiology score (SAPS II), disease severity, calculated from 12 routine physiological measurements made during the first $24 \mathrm{~h}$ with information about previous health status obtained at admission [12]. Each parameter was monitored at admission and then at least once per week, and we recorded the most abnormal value for each parameter each week. Infection was diagnosed if microorganisms were isolated or if clinical suspicion was high, based on clinical, biological and radiological symptoms. For suspected pneumonia, microbiological confirmation of respiratory sampling using bronchoscopy was not performed because of the high associated risks in these patients.

\section{Statistical analysis}

Statistical analysis was performed using Statview 4.5 software. Results are expressed as median (min-max). Categorical variables were compared using Chi-squared and Fisher's exact test as appropriate. Quantitative variables were compared using Mann-Whitney analysis. Changes of recorded variables over time were evaluated by one-way ANOVA for repeated measurements. The Kaplan-Meier method was used to estimate survival status and the log-rank test was used for survival distribution comparison. A p-value $<0.05$ was considered statistically significant.

\section{RESULTS}

\section{Baseline characteristics of the patients}

46 patients were included (table 1). Median (min-max) age at admission was 50.0 (16.2-77.4) years, with a female/male ratio of 2.3/1 (31 females). Median body mass index was 20.9 (15.9$33.6) \mathrm{kg} \cdot \mathrm{m}^{-2} .23$ patients were admitted in ICU directly from the respiratory ward of our institution, 15 were referred from another hospital, six were referred from the emergency department of our institution and two came directly from home. Average time from PAH diagnosis to ICU admission was 6.3 (2.3-16.5) years. The patients suffered from idiopathic PAH $(n=24)$, inoperable chronic thromboembolic pulmonary hypertension (CTEPH; $n=7)$, systemic sclerosis-associated PAH $(n=5), P A H$ associated with connective tissue diseases other than scleroderma $(n=2)$, HIV-related PAH $(n=3)$, portopulmonary hypertension $(n=3)$ and $\mathrm{PAH}$ associated with congenital heart disease $(n=2)$. Specific pulmonary hypertension therapy at admission included intravenous prostacyclin alone $(n=9)$, intravenous prostacyclin in combination with bosentan $(n=10)$ or with sildenafil $(n=1)$, combination of intravenous prostacyclin with bosentan and sildenafil $(n=3)$, bosentan alone $(n=10)$ or in combination with sildenafil $(n=7)$ 
or inhaled iloprost $(n=2)$, subcutaneous treprostinil $(n=1)$ or no specific treatment $(n=3)$. No difference was found in baseline characteristics between patients who died in ICU versus patients discharged from ICU, although there was a nonsignificant trend towards older age at admission in ICU and a more severe baseline haemodynamic status among nonsurvivors (table 2).

\section{Survival}

The mean (range) ICU length of stay was 14 (1-87) days. Overall mortality in ICU was $41.3 \%$ (fig. 1 ). There was no difference in terms of time to discharge or death according to outcome in ICU. Six out of the 27 patients discharged from ICU were dead at 3 months, giving a 3-month mortality of $22.2 \%$ for patients discharged from ICU. Among patients discharged from ICU, seven were subsequently referred at least a second time to the ICU for similar symptoms.

\section{Clinical and biochemical data at admission in ICU}

There was no clinical difference between survivors and nonsurvivors on admission to the ICU. No difference was found in terms of diuresis, but it is of note that the furosemide level to achieve a diuresis on the first day was higher in patients with an unfavourable outcome. Triggering factors for PAH decompensation were identified in 19 patients, as follows: unplanned modification or withdrawal of pulmonary vasodilator therapy $(n=3)$; unplanned withdrawal of diuretics

\begin{tabular}{|c|c|c|}
\hline TABLE 1 & $\begin{array}{l}\text { Baseline characteristics of } \\
\text { the patients }\end{array}$ & \\
\hline \multicolumn{2}{|l|}{ Age yrs } & $50.0(16.2-77.4)$ \\
\hline \multicolumn{2}{|l|}{ Sex F/M n } & $2.3 / 1$ \\
\hline \multicolumn{2}{|l|}{$\mathrm{BMI} \mathbf{k g} \cdot \mathrm{m}^{-2}$} & $20.9(15.9-33.6)$ \\
\hline \multicolumn{3}{|c|}{ Type of PAH $n$} \\
\hline \multicolumn{2}{|c|}{ Idiopathic PAH } & 24 \\
\hline \multicolumn{2}{|c|}{ Inoperable CTEPH } & 7 \\
\hline \multicolumn{2}{|c|}{ Systemic sclerosis-associated PAH } & 5 \\
\hline \multicolumn{2}{|c|}{$\begin{array}{l}\text { PAH associated with connective tissue diseases } \\
\text { other than scleroderma }\end{array}$} & 2 \\
\hline \multicolumn{2}{|c|}{ HIV-related PAH } & 3 \\
\hline \multicolumn{2}{|c|}{ Portopulmonary hypertension } & 3 \\
\hline \multicolumn{2}{|c|}{ PAH associated with congenital heart disease } & 2 \\
\hline \multicolumn{3}{|c|}{ NYHA functional class when last stable $n$} \\
\hline \multicolumn{2}{|c|}{$\|$} & 5 \\
\hline \multicolumn{2}{|l|}{ III } & 26 \\
\hline \multicolumn{2}{|c|}{ IV } & 15 \\
\hline \multicolumn{3}{|c|}{ Pulmonary haemodynamic data when last stable } \\
\hline \multicolumn{2}{|c|}{$\bar{P}$ pa mmHg } & $52(32-103)$ \\
\hline \multicolumn{2}{|c|}{ PVR dyn $\cdot \mathrm{s} \cdot \mathrm{cm}^{-5}$} & $1016(525-2400)$ \\
\hline \multicolumn{2}{|c|}{ Pra $\mathrm{mmHg}$} & $12(0-32)$ \\
\hline \multicolumn{2}{|c|}{$\mathrm{Cl} \mathrm{L} \cdot \mathrm{min}^{-1} \cdot \mathrm{m}^{-2}$} & $2.23(1.47-5.0)$ \\
\hline \multicolumn{2}{|l|}{ SAPS II } & $24(6-49)$ \\
\hline
\end{tabular}

Data are presented as median (min-max), unless otherwise stated. F: female; M: male; BMI: body mass index; PAH: pulmonary arterial hypertension; CTEPH: chronic thromboembolic pulmonary hypertension; NYHA: New York Heart Association; $\bar{P}$ pa: mean pulmonary arterial pressure; PVR: pulmonary vascular resistance; Pra: right atrial pressure; Cl: cardiac index; SAPS II: simplified acute physiology score II. $(n=1)$; septicaemia $(n=5)$; pneumonia $(n=3)$; purulent pleural effusion $(n=1)$; septic state without bacterial documentation $(n=2)$; cardiac arrhythmia $(n=3)$; and unplanned pregnancy $(n=1)$. There was no difference in outcome according to the identification of a triggering factor. At admission, median serum level of BNP was $1415(449-3550) \mathrm{pg} \cdot \mathrm{mL}^{-1}$ in nonsurvivors versus $628 \quad(87-1460) \mathrm{pg} \cdot \mathrm{mL}^{-1}$ in survivors $(p=0.0007)$. Serum creatinine was also increased in nonsurvivors compared with survivors 112 (42-144) versus 95 (82151) $\mu \mathrm{mol} \cdot \mathrm{L}^{-1}$, respectively $(\mathrm{p}=0.04)$, and median CRP level was $40(0-270)$ versus $12(0-200) \mathrm{mg} \cdot \mathrm{L}^{-1}$, respectively $(\mathrm{p}=0.01)$ (table 3). We performed a logistic regression with three variables considering the small sample of our cohort (CRP, creatinine and $\mathrm{BNP}$ ) and found that BNP was the only independent predictor of mortality $(p=0.005,95 \%$ CI $1.001-$ 1.004). No difference in troponin Ic or serum sodium was found between survivors and nonsurvivors. CRP serum levels at admission were associated with evidence of an infectious episode irrespective of patients clinical course (16 (0-197) $\mathrm{mg} \cdot \mathrm{dL}^{-1}$ if no infection was found versus $92(0-277) \mathrm{mg} \cdot \mathrm{dL}^{-1}$ with confirmed infection; $\mathrm{p}=0.01)$. We also found a significant association between SAPS II and the clinical course in ICU (32 (11-49) in nonsurvivors versus 22 (6-43) in survivors; $\mathrm{p}=0.001)$.

\section{Clinical and biochemical data during hospitalisation according to survival in ICU}

During the first 3 weeks of hospitalisation, the only clinical parameter associated with survival was systemic arterial pressure (systolic and mean systemic arterial pressure, ANOVA for repeated measurements: $p=0.003$ and $p<0.0001$, respectively). Biochemical parameters associated with outcome included serum levels of $\mathrm{BNP}, \mathrm{CRP}$, creatinine and sodium. ANOVA for repeated measurements: $p<0.0001$ for all variables. (fig. 2)

The occurrence of an infection during ICU hospitalisation was associated with a worse outcome, with 14 out of 19 of the nonsurvivors exhibiting an in hospital acquired infection compared with six out of the 27 survivors $(p=0.0005)$

\section{Treatments during ICU stay according to survival}

Changes in dobutamine doses over time were associated with ICU survival; progressive increase in dobutamine administration rate was associated with poorer outcome (fig. 3). Changes in norepinephrine dose over time were no different between survivors and nonsurvivors. Continuous intravenous prostacyclin was administered 15 times, in a nonrandomised fashion, twice in combination with bosentan, and continuous nitric oxide inhalation was used 13 times. These treatments were given to patients whose severity was considered very high and who were not already receiving these treatments as background PAH therapy. Neither of these treatments significantly influenced survival, although this study was not designed or powered to evaluate this association with outcome.

\section{DISCUSSION}

This is the first prospective study analysing consecutive patients requiring ICU admission for severe acute right ventricular failure due to decompensated PAH. We found a very high overall ICU mortality $(41.3 \%)$, which was predicted by high admission BNP levels. Patients who had higher 
TABLE 2 Baseline characteristics of patients according to survival in the intensive care unit

\begin{tabular}{|c|c|c|c|}
\hline Subjects $n$ & 19 & 27 & \\
\hline Sex F/M n & $2.3 / 1$ & $2.2 / 1$ & 0.7 \\
\hline BMI $\mathbf{k g} \cdot \mathrm{m}^{-2}$ & $21.7(16.7-30.8)$ & $20.3(15.9-33.6)$ & 0.7 \\
\hline \multicolumn{4}{|l|}{ Type of РАH $n$} \\
\hline Systemic sclerosis-associated PAH & 4 & 1 & ND \\
\hline PAH associated with connective tissue diseases other than scleroderma & 1 & 1 & ND \\
\hline HIV-related PAH & 1 & 2 & ND \\
\hline Portopulmonary hypertension & 1 & 2 & ND \\
\hline PAH associated with congenital heart disease & 1 & 1 & ND \\
\hline \multicolumn{4}{|l|}{ Pulmonary haemodynamic data when last stable } \\
\hline $\bar{P}$ pa $\mathrm{mmHg}$ & $52(32-87)$ & $52(40-103)$ & 0.6 \\
\hline PVR dyn.s. $\mathrm{cm}^{-5}$ & $1054(623-2400)$ & $990(525-2315)$ & 0.5 \\
\hline Pra mmHg & $12(3-32)$ & $10(0-21)$ & 0.7 \\
\hline $\mathrm{Cl} \mathrm{L} \cdot \mathrm{min}^{-1} \cdot \mathrm{m}^{-2}$ & $2.14(1.48-3.10)$ & $2.26(1.47-5.0)$ & 0.4 \\
\hline
\end{tabular}

baseline serum creatinine and CRP levels, and those receiving higher baseline doses of oral furosemide also did worse. The presence of a better pre-ICU functional class did not influence ICU outcome. Nonsurvivors required much higher doses of dobutamine.

There has been only one other recent retrospective study of PAH patients, where $27 \%$ of episodes of decompensated RVF were precipitated by infection, $48 \%$ had no obvious precipitant
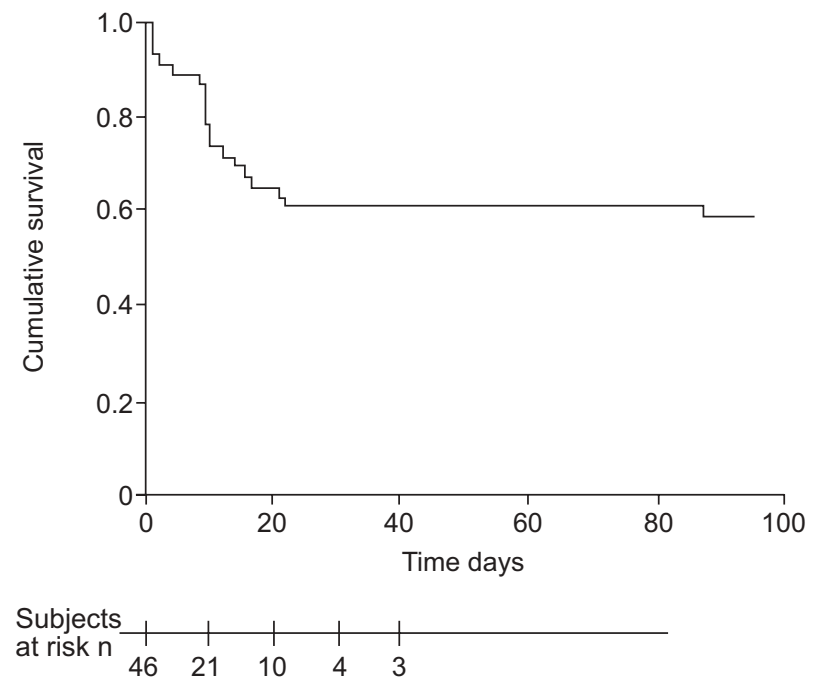

FIGURE 1. Survival in overall population. (so were presumed due to disease progression) and overall mortality was high, especially in those with infection-related episodes (50\%) and in "cold-dry" haemodynamic profiles (at $100 \%$ ). Similarly, the presence of a triggering factor made no impact on outcome [13]. It has been recently emphasised that we lack an evidence-based consensus for managing acute RV failure in these patients [5], which are difficult to manage, despite the fact that, as in acute left ventricular failure [14], these episodes account for significant patient mortality.

BNP and NT-proBNP have been shown to be associated with long-term outcome in stable PAH [15, 16], and are useful biomarkers of RV dysfunction in patients with acute pulmonary embolism, in the setting of acute RV pressure overload [17, 18]. This is the first study to test the prognostic significance of absolute value of BNP in the setting of acute right heart failure in decompensated PAH. BNP is secreted by cardiac ventricles trough a constitutive pathway and is increased according to the degree of myocardial stretch, damage and ischaemia. Previous work has shown an increase in BNP mRNA expression as early as the first day following pulmonary artery coarctation in rats [19]. Despite the absence of right heart catheterisation at admission in our cohort, resulting in uncertainties concerning the fluctuations of haemodynamic values during worsening, our results suggest that BNP can be used as a prognostic marker in these patients. NT-proBNP has also been shown to be a valuable prognostic marker in stable $\mathrm{PAH}$ [20]; however, since it is only excreted renally without prior degradation, its accuracy in patients with possible renal failure might be diminished [21]. 
TABLE 3 Clinical and biochemical data at admission according to survival in the intensive care unit

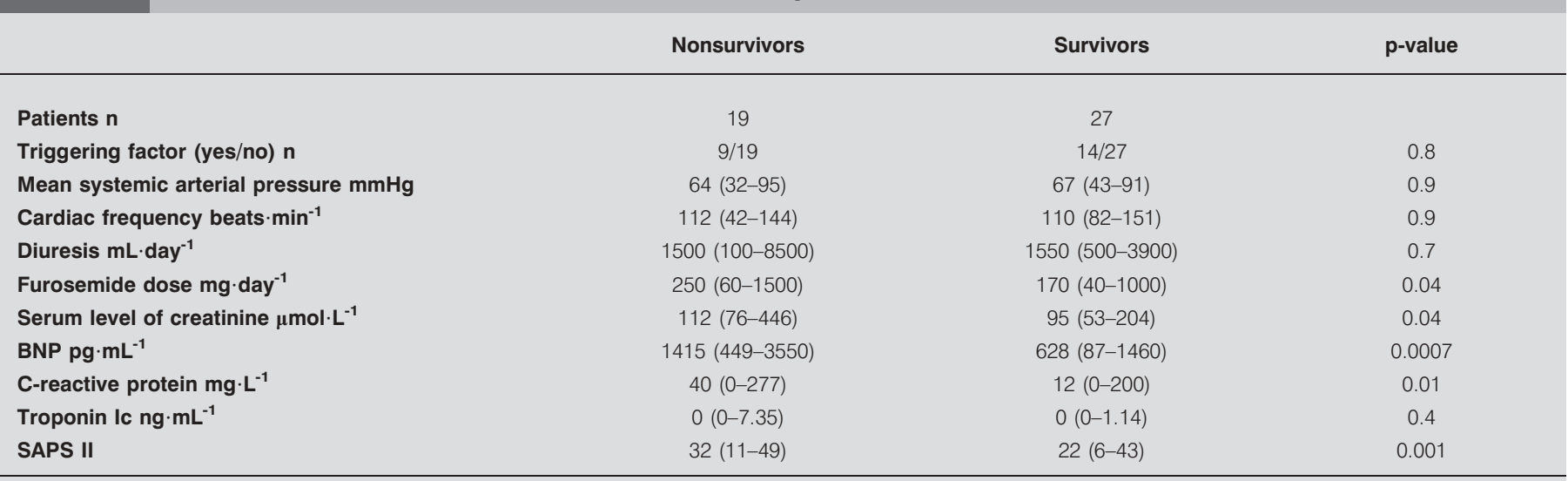

BNP: brain natriuretic peptide; SAPS II: simplified acute physiology score II.

Troponin has been shown to be associated with prognosis in PAH when evaluated on a long-term basis [22]. RV dysfunction in acute pulmonary embolism is also associated with increased levels of troponin $[23,24]$. Nevertheless, despite the difference in BNP results between survivors and nonsurvivors, our results for troponin did not show a statistical link with survival in the setting of acutely worsened PAH. This was a similar finding in the study by KURZYNA et al. [13]. Whether our sample of patients, our dosage technique sensitivity or the physiology of ventricular injury may explain this absence of link remains to be further investigated. In future studies the most sensitive assays for troponin measurements should be used.
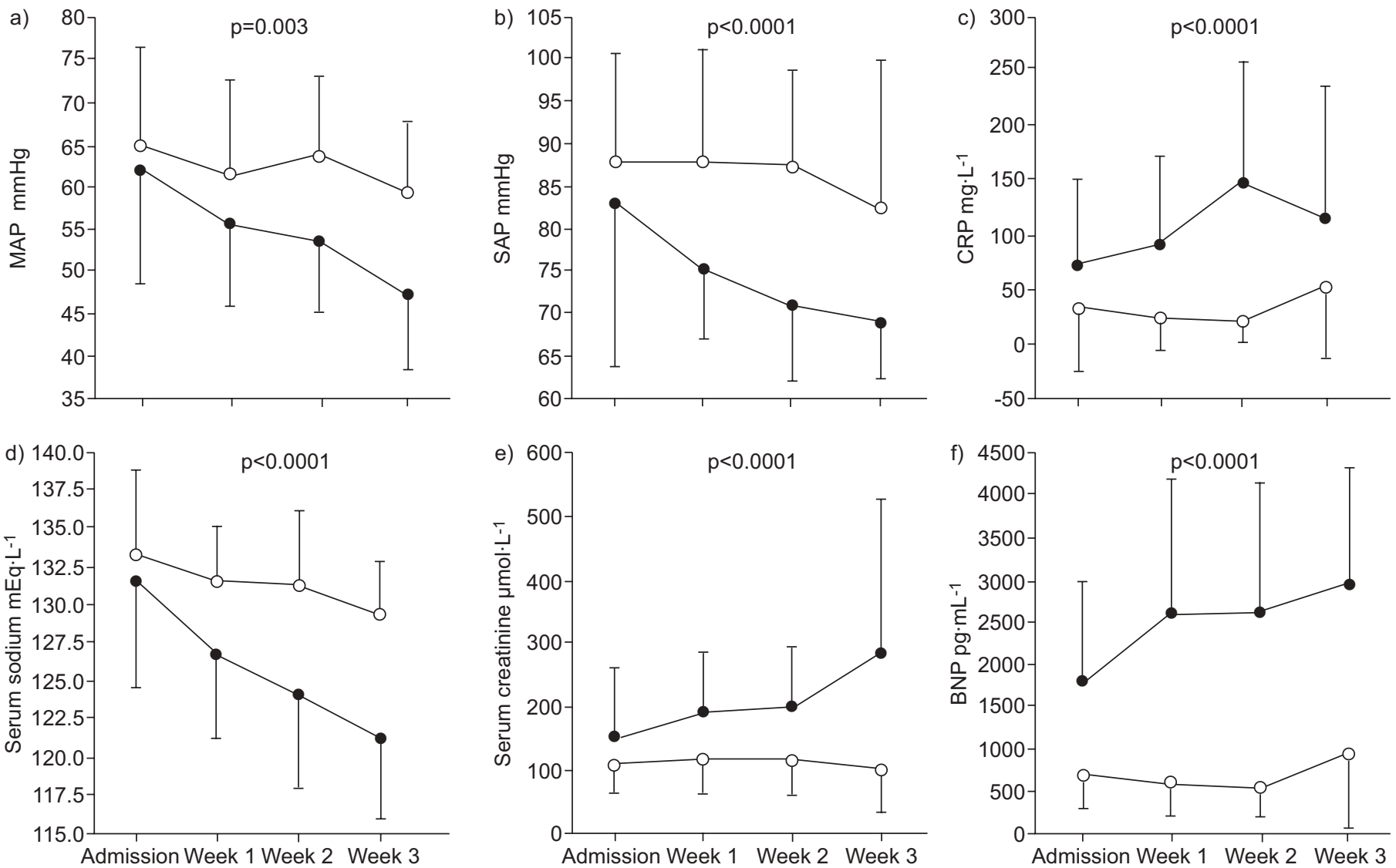

FIGURE 2. Clinical and biochemical data during intensive care unit (ICU) stay according to survival in ICU: patients discharged from the ICU (survivors; $O$ ) or patients who died in the ICU (nonsurvivors; $\bullet$ ). a) Changes in mean systemic arterial pressure (MAP), b) systolic systemic arterial pressure (SAP), c) C-reactive Protein (CRP) serum levels, d) sodium serum levels, e) creatinine serum levels, and f) brain natriuretic peptide (BNP) serum levels, during ICU stay. 


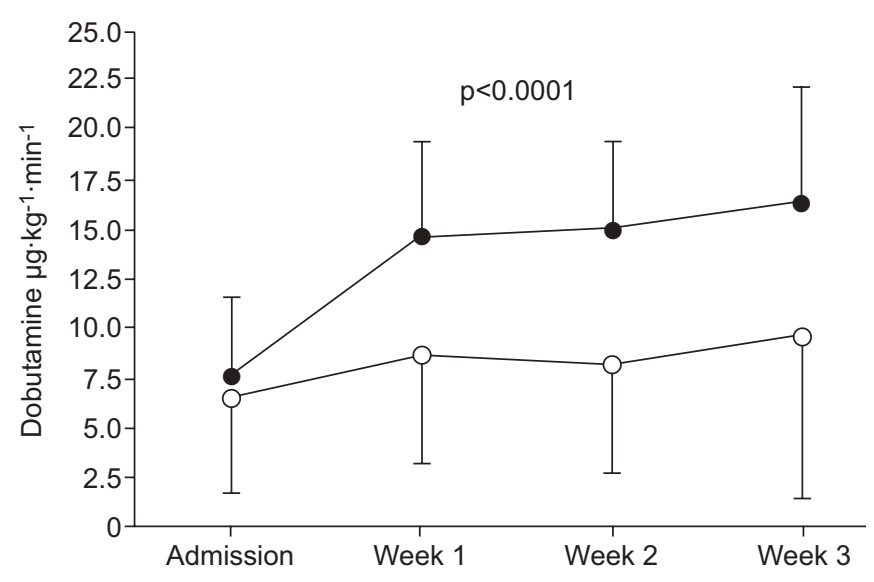

FIGURE 3. Changes in dobutamine doses according to survival in the intensive care unit (ICU): patients discharged from the ICU (survivors; $\bigcirc$ ) or patients who died in ICU (nonsurvivors;

The concept of rapidly introducing a specific PAH therapy in ICU in these patients remains questionable, and some concern exists about possible unfavourable systemic haemodynamic effects with these treatments, which presumably act, at least in part, through an antiproliferative and a vasodilatory effect on a long-term basis [25]. Experimental animal data in a model of acute load-induced right ventricular failure have indicated that intravenous prostacyclin improves RV to pulmonary artery coupling and cardiac output [26]. The use of specific PAH treatment was not associated with improved survival in our study. In the absence of a comparator and a systematic randomisation of patients to receive specific $\mathrm{PAH}$ therapy or not, one cannot make conclusions regarding the benefit of initiating such therapies in the ICU. Given the absence of precise guidelines in this setting, we introduced novel PAH therapy in the most severe patients, using intravenous epoprostenol as the best evidenced vasodilatory drug in NYHA class IV patients $[27,28]$. This might have biased our analysis in favour of the less severe patients. Furthermore, as we did not perform right-heart catheterisation, we cannot discuss the haemodynamic effects of such rescue-"specific" PAH treatment in our patients. Thus, no conclusions can be drawn based on the present study on that point and we suggest that more studies are urgently needed. Further to the lack of benefit from these available medical therapies, our data demonstrate the poor prognosis of acute heart failure in patients with $\mathrm{PAH}$, emphasising the need to consider novel therapeutic approaches in these subjects, such as listing for urgent lung transplantation and the possible use of RV assistance devices $[29,30]$.

Renal impairment and water regulation imbalance have been extensively studied in left ventricular failure [31], however no study has addressed this in acute right-heart failure, complicating PAH. Recent data suggest that not only cardiac output but also right atrial pressure in pulmonary hypertension may contribute to the complex pathophysiological network including rennin-angiotensin-aldosterone, natriuretic peptides, vasopressin and sympathetic nervous systems, resulting in the observed metabolic abnormalities [32]. In left-heart disease, data suggest that these abnormalities are linked to survival [33], and it has been recently found that hyponatraemia is predictive of poor survival for PAH patients in stable condition. The pathophysiological mechanisms have been proposed to mirror those in left-heart failure [34]. Some studies have tested the efficacy of specific therapeutic interventions antagonising the vasopressin pathway in left-heart failure (outside the ICU setting), showing an improvement in serum level of sodium and dyspnoea, but no change in survival $[35,36]$. We also found a link between survival and changes of serum level of sodium during follow-up, reinforcing the importance of its measurement in this setting. A recent study has also provided evidence that the serum level of creatinine is associated with survival in patients with stable $\mathrm{PAH}$, although the pathophysiological mechanism has not yet been elucidated [37].

It is of note that CRP serum levels in patients without identified infection at admission were also slightly elevated; even though statistically lower than in patients with a documented infection. A very recent study underlined the value in predicting outcome and response to therapy of this marker in stable PAH [38]. This raises the question about the involvement of inflammatory mechanisms whose origin and nature remain elusive but are being studied in the pathophysiology of chronic PAH $[39,40]$. Such inflammatory pathways stress the need for more studies on inflammation in both stable and decompensated $\mathrm{PAH}$, in order to identify novel therapeutic targets. Infection caries a very bad prognosis in $\mathrm{PAH}$ patients referred for acute heart failure. In sepsis, both experimental models and clinical examples support the notion that both right- and left-ventricular injury is relatively common, leading to primary myocardial dysfunction [41]. The poor prognosis associated with the occurrence of an infection in ICU, therefore, underlines the need for efficient preventive, diagnostic and prompt treatment strategies in $\mathrm{PAH}$ patients admitted in ICU with suspected infection.

Our study has limitations that have to be acknowledged. First, this was a single-centre study; however, our National Reference Centre for Pulmonary Hypertension is one of the largest pulmonary vascular centre worldwide, which recruits $>50 \%$ of French patients treated for PAH [42]. In addition, it is one of the very few centres with an ICU devoted to the care of pulmonary hypertensive patients. Therefore, this single-centre characteristic may also have the advantage of allowing recruitment of consecutive $\mathrm{PAH}$ patients with a similar management approach performed by the same team. Secondly, our cohort was rather small $(n=46)$ and was limited to patients with severe RV failure requiring catecholamine. Considering that the main objective of the study was to describe the clinical course of PAH patients in the setting of primary acute circulatory failure, this characteristic may prevent the extrapolation of our findings to the other subgroups. Nevertheless, PAH is a rare condition with prevalence ranging from 5 to 25 cases per million in France [42], highlighting the major difficulty in obtaining large numbers of patients for clinical studies. Also, some of the parameters could not be recorded every week of the study thus limiting the analysis of the true variability of each factor during the entire study period. Despite this, our data are sufficiently powered to determine the clinical and laboratorial parameters associated with poor prognosis during ICU admission. Finally, the lack of invasive haemodynamic monitoring is a weakness 
that may have meant that we missed important pathophysiological parameters and potential prognostic factors. Nevertheless, invasive haemodynamic monitoring may be hazardous in severe unstable PAH patients. Further studies should include at least echocardiographic parameters already validated as prognostic factors in stable PAH patients, such as heart chamber size, systolic pulmonary artery pressure, pericardial effusion and/or tricuspid annular plane systolic excursion index $[43,44]$. Although echocardiography may provide important data on systolic pulmonary arterial pressure and cardiac index, appropriate "right ventricular preload" and the way to monitor it safely on a regular basis needs to be determined in further studies.

In conclusion, we have described the first prospective cohort of PAH admitted in ICU due to acute right-heart failure. ICU and 3-month mortality rates found in our study confirmed the dismal prognosis of such episodes. Some simple clinical and biological variables appear to be strongly linked with the clinical course in ICU and these findings stress the need for further studies in order to propose future recommendations in this devastating complication of PAH.

\section{STATEMENT OF INTEREST}

Statements of interest for X. Jaïs, O. Stibon, G. Simonneau and M. Humbert can be found at www.erj.ersjournals.com/misc/ statements.dtl

\section{REFERENCES}

1 Chin KM, Rubin LJ. Pulmonary arterial hypertension. J Am Coll Cardiol 2008; 51: 1527-1538.

2 Galie N, Rubin L. Pulmonary arterial hypertension. Epidemiology, pathobiology, assessment and therapy. J Am Coll Cardiol 2004; 43: S1-S90.

3 Kearon C, Kahn SR, Agnelli G, et al. Antithrombotic therapy for thromboembolic disease. Chest 2008; 133: 454S-545S

4 McNeil K, Dunning J, Morrell NW. The pulmonary physician in critical care. 13: the pulmonary circulation and right ventricular failure in the ITU. Thorax 2003; 58: 157-162.

5 Zamanian RT, Haddad F, Doyle RL, et al. Management strategies for patients with pulmonary hypertension in the intensive care unit. Crit Care Med 2007; 35: 2037-2050.

6 Piazza G, Goldhaber SZ. The acutely decompensated right ventricle. Chest 2005; 128: 1836-1852.

7 Kerbaul F, Rondelet P, Demester JP, et al. Effects of levosimendan versus dobutamine on pressure load-induced right ventricular failure. Crit Care Med 2006; 34: 2814-2819.

8 Leier CV, Heban PT, Huss P, et al. Comparative systemic and regional hemodynamic effects of dopamine and dobutamine in patients with cardiomyopathic heart failure. Circulation 1978; 58: 466-475.

9 Kerbaul F, Rondelet B, Motte S, et al. Effects of norepinephrine and dobutamine on pressure-load induced right ventricular failure. Crit Care Med 2004; 32: 1035-1040.

10 Rattes M, Calvin JE. Acute pulmonary hypertension. In: Pinsky MR, Dhainaut JF, eds. Pathophysiologic Foundations of Critical Care. Baltimore, Williams and Wilkins, 1993; pp. 312-336.

11 Sztrymf B, Humbert M. Practical management of pulmonary arterial hypertension in the intensive care unit. In: Humbert M, Lynch III JP, eds. Pulmonary Hypertension. Informa Heathcare USA, New York, 2009; pp. 455-461.

12 Le Gall JR, Lemeshow S, Saulnier F. A new Simplified Acute Physiology Score (SAPS II) based on a European/North American multicenter study. JAMA 1993; 270: 2957-2963.
13 Kurzyna M, Zyłkowska J, Fijałkowska A, et al. Characteristics and prognosis of patients with decompensated right ventricular failure during the course of pulmonary hypertension. Kardiol Pol 2008; 66: 1033-1039.

14 Nieminen MS, Brutsaert D, Dickstein K, et al. EuroHeart Failure Survey II (EHFS II): a survey on hospitalized acute heart failure patients: description of population. Eur Heart J 2006; 27: 2725-2736.

15 Nagaya N, Nishikimi T, Uematsu M, et al. Plasma brain natriuretic peptide as a prognostic indicator in patients with primary pulmonary hypertension. Circulation 2000; 102: 865-870.

16 Fijalkowska A, Kurzyna M, Torbicki A, et al. Serum N-terminal Brain ntriuretic peptide as a prognostic parameter in patients with pulmonary hypertension. Chest 2006; 129: 1313-1321.

17 Kucher N, Printzen G, Goldhaber SZ. Prognostic role of brain natriuretic peptide in acute pulmonary embolism. Circulation 2003; 107: 2545-2547.

18 Pieralli F, Olivotto I, Vanni S, et al. Usefulness of bedside testing for brain natriuretic peptide to identifie right ventricular dysfunction and outcome in normotensive patients with acute pulmonary embolism. Am J Cardiol 2006; 97: 1386-1390.

19 Adachi S, Ito H, Ohta $\mathrm{Y}$, et al. Distribution of mRNAs natriuretic peptides in right ventricular hypertrophy after pulmonary arterial banding. Am J Physiol 1995; 268: H162-H169.

20 Souza R, Bogossian HB, Humbert M, et al. N-terminal-pro-brain natriuretic peptide as a haemodynamic marker in idiopathic pulmonary arterial hypertension. Eur Respir J 2005; 25: 509-513.

21 Leuchte $\mathrm{HH}$, El Nounou M, Tuerpe JC, et al. N-terminal pro-brain natriuretic peptide and renal insufficiency as predictors of mortality in pulmonary hypertension. Chest 2007; 131: 401-409.

22 Torbicki A, Kurzyna M, Kuca P, et al. Detectable cardiac troponin T as a marker of poor prognosis among patients with chronic precapillary pulmonary hypertension. Circulation 2003; 108: 844-848.

23 Meyer T, Binder L, Hruska N, et al. Cardiac troponin I elevation in acute pulmonary embolism is associated with right ventricular dysfunction. J Am Coll Cardiol 2000; 36: 1632-1636.

24 Mehta NJ, Jani K, Khan IA. Clinical usefulness and prognostic value of elevated cardiac troponin I levels in acute pulmonary embolism. Am Heart J 2003; 145: 821-825.

25 Humbert M, Sitbon O, Simonneau G. Treatment of pulmonary arterial hypertension. N Engl J Med 2004; 351: 1425-1436.

26 Kerbaul F, Brimioulle S, Rondelet B, et al. How prostacyclin improves cardiac output in right heart failure in conjunction with pulmonary hypertension. Am J Respir Crit Care Med. 2007; 175: 846-850.

27 Galiè N, Hoeper MM, Humbert M, et al. Guidelines for the diagnosis and treatment of pulmonary hypertension. The Task Force for the Diagnosis and Treatment of Pulmonary Hypertension of the European Society of Cardiology (ESC) and the European Respiratory Society (ERS), endorsed by the International Society of Heart and Lung Transplantation (ISHLT). Eur Respir J 2009; 34: $1219-1263$.

28 Galiè N, Hoeper MM, Humbert M, et al. Guidelines for the diagnosis and treatment of pulmonary hypertension: The Task Force for the Diagnosis and Treatment of Pulmonary Hypertension of the European Society of Cardiology (ESC) and the European Respiratory Society (ERS), endorsed by the International Society of Heart and Lung Transplantation (ISHLT). Eur Heart J 2009; 30: 2493-2537.

29 Pedemonte VO, Aránguiz Santander E, Torres H H, et al. [Right ventricular assistance with a centrifugal pump. Report of two cases]. Rev Mal Chil 2008; 136: 359-366.

30 Montani D, Jaïs X, Price LC, et al. Cautious use of epoprostenol therapy is a safe bridge to lung transplantation in pulmonary veno-occlusive disease. Eur Respir J 2009; 34: 1348-1356.

31 Schrier RW. Water and sodium retention in oedematous disorders: role of vasopressin and aldosteron. Am J Med 2006; 119: S47-S53. 
32 Damman K, Navis G, Smilde T, et al. Decreased cardiac output, venous congestion and the association with renal impairment in patients with cardiac dysfunction. Eur J Heart Fail 2007; 9: 872-878.

33 Gheorghiade M, Abraham WT, Albert NM, et al. Relationship between admission serum sodium and outcome in patients hospitalized for heart failure: an analysis from the OPTIMIZEHF registry. Eur Heart J 2007; 28: 980-988.

34 Forfia PR, Mathai SC, Fisher MR, et al. Hyponatremia predicts right heart failure and poor survival in pulmonary arterial hypertension. Am J Respir Crit Care Med 2008; 177: 1364-1369.

35 Konstam MA, Gheorghiade M, Burnett JC Jr, et al. Effects of oral Tolvaptan in patients hospitalized for worsening heart failure: the EVEREST outcome trial. JAMA 2007; 297: 1319-1331.

36 Gheorghiade M, Niazi I, Ouyang J, et al. Vasopressin V2-receptor blockade with tolvaptan in patients with chronic heart failure. Results from a double blind, randomized trial. Circulation 2003; 107: 2690-2696.

37 Shah SJ, Thenappan T, Rich S, et al. Association of serum creatinine with hemodynamics and mortality in pulmonary arterial hypertension. Circulation 2008; 117: 2475-2483.
38 Quark M, Nawrot T, Meyns B, et al. C-reactive protein: a new predictor of adverse outcome in pulmonary arterial hypertension. J Am Coll Cardiol 2009; 53: 1211-1218.

39 Dorfmuller P, Perros F, Balabanian K, et al. Inflammation in pulmonary arterial hypertension. Eur Respir J 2003; 22: 358-363.

40 Humbert M, Morrel NW, Archer SL, et al. Cellular and molecular pathobiology of pulmonary arterial hypertension. J Am Coll Cardiol 2004; 43: S13-S24.

41 Sharma AC. Sepsis-induced myocardial dysfunction. Shock 2007 28: 265-269.

42 Humbert M, Sitbon O, Chaouat A, et al. Pulmonary arterial hypertension in France: results from a national registry. Am J Respir Crit Care Med 2006; 173: 1023-1030.

43 Forfia PR, Fisher MR, Mathai SC, et al. Tricuspid annular displacement predicts survival in pulmonary hypertension. Am J Respir Crit Care Med 2006; 174: 1034-1041.

44 Bossone E, Bodini BD, Mazza A, et al. Pulmonary arterial hypertension: the key role of echocardiography. Chest 2005; 127: 1836-1843. 